\title{
Lifetime and Performance Prediction of SOFC Anodes Operated with Trace Amounts of Hydrogen Sulfide
}

\author{
Matthias Riegraf, Günter Schiller, Rémi Costa, \\ K. Andreas Friedrich, Arnulf Latz, Vitaliy Yurkiv \\ German Aerospace Center (DLR), Stuttgart, Germany \\ 30.07.2015
}

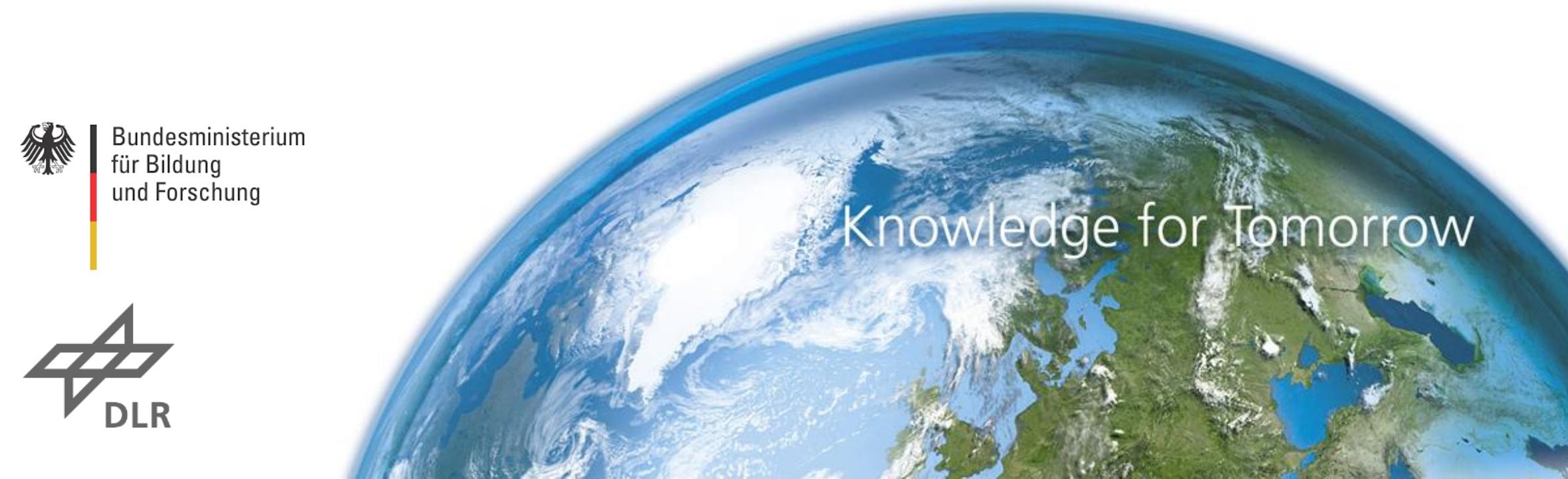




\section{Overview}

$>$ Motivation and aim of the work

$>$ Computational methods

$>$ Results

- Reaction mechanism development

- Sulfur poisoning in $\mathrm{H}_{2} / \mathrm{H}_{2} \mathrm{O}$ fuels

- Sulfur poisoning of internal methane steam reforming

$>$ Summary and conclusions 


\section{Overview}

$>$ Motivation and aim of the work

$>$ Computational methods

$>$ Results

- Reaction mechanism development

- Sulfur poisoning in $\mathrm{H}_{2} / \mathrm{H}_{2} \mathrm{O}$ fuels

- Sulfur poisoning of internal methane steam reforming

$>$ Summary and conclusions 


\section{Motivation and aim of the work}

- Ni/YSZ anode poisoning with $\mathrm{H}_{2} \mathrm{~S}$

- Despite extensive experimental and theoretical investigation, still no validated mechanism for sulfur poisoning

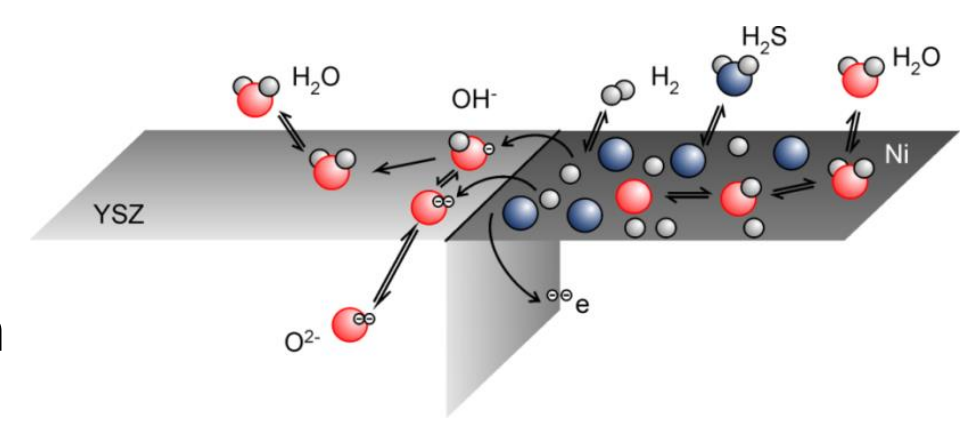

- Identification of underlying processes with elementary kinetic modeling

- Derivation of thermodynamic and kinetic data

Goal: $>$ Building of a reaction mechanism and identification of the elementary processes leading to sulfur poisoning of Ni/YSZ anodes 


\section{Overview}

$>$ Motivation and aim of the work

$>$ Computational methods

$>$ Results

- Reaction mechanism development

- Sulfur poisoning in $\mathrm{H}_{2} / \mathrm{H}_{2} \mathrm{O}$ fuels

- Sulfur poisoning of internal methane steam reforming

$>$ Summary and conclusions 


\section{Computational methods: Elementary kinetic modeling}

\section{framework}

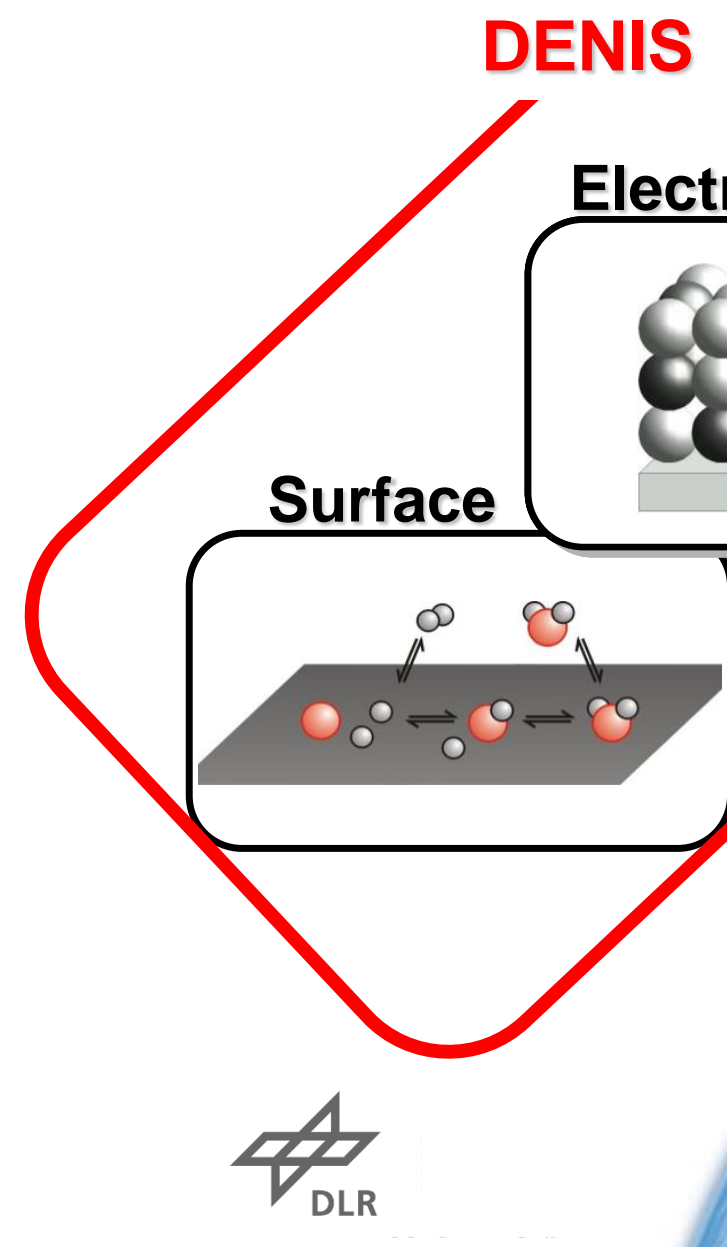

DENIS: Detailed Electrochemistry and Numerical Impedance Simulation

- Heterogeneous and Mean-field approach, electrochemistry: elementary kinetic description of reactions

- Porous phase transport:

Fickian, Knudsen diffusion and Darcy flow

- Channel flow: Navier-Stokes equations 


\section{Overview}

$>$ Motivation and aim of the work

$>$ Computational methods

$>$ Results

- Reaction mechanism development

- Sulfur poisoning in $\mathrm{H}_{2} / \mathrm{H}_{2} \mathrm{O}$ fuels

- Sulfur poisoning of internal methane steam reforming

$>$ Summary and conclusions 


\section{Reaction mechanism development}

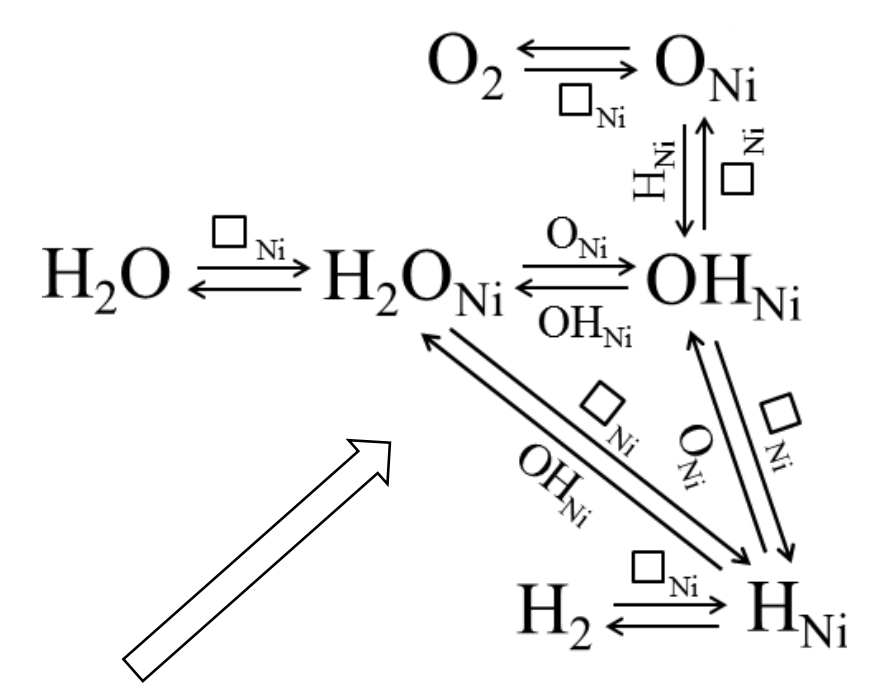

- $\mathrm{H}_{2}$ oxidation mechanism validated in previous modeling studies $[1,2]$

- Assumed to be unaffected by the presence of sulfur
$\mathrm{S}$ formation/oxidation at $\mathrm{Ni}$ surface:

$$
\begin{aligned}
& \mathrm{H}_{2} \mathrm{~S}_{\mathrm{g}}+\square_{\mathrm{Ni}} \rightleftharpoons \mathrm{H}_{2} \mathrm{~S}_{\mathrm{Ni}} \\
& \mathrm{H}_{2} \mathrm{~S}_{\mathrm{Ni}}+\square_{\mathrm{Ni}} \rightleftharpoons \mathrm{HS}_{\mathrm{Ni}}+\mathrm{H}_{\mathrm{Ni}} \\
& \mathrm{HS}_{\mathrm{Ni}}+\square_{\mathrm{Ni}} \rightleftharpoons \mathrm{H}_{\mathrm{Ni}}+\mathrm{S}_{\mathrm{Ni}} \\
& \mathrm{S}_{\mathrm{Ni}}+\mathrm{O}_{\mathrm{Ni}} \rightleftharpoons \mathrm{SO}_{\mathrm{Ni}}+\square_{\mathrm{Ni}} \\
& \mathrm{SO}_{\mathrm{Ni}}+\mathrm{O}_{\mathrm{Ni}} \rightleftharpoons \mathrm{SO}_{2, \mathrm{Ni}}+\square_{\mathrm{Ni}} \\
& \mathrm{SO}_{2, \mathrm{Ni}} \rightleftharpoons \mathrm{SO}_{2,9}+\square_{\mathrm{Ni}} \\
& \text { - } \text { Extension of original } \\
& \text { reaction mechanism }
\end{aligned}
$$

- Present work: Derivation of thermodynamic and kinetic data 


\section{Reaction mechanism development: Data derivation}

Thermodynamic and kinetic data from literature:

- Derivation from DFT calculations [1-3]

- Entropy of $\mathrm{S}_{\mathrm{Ni}}$ is constant: $s\left(\mathrm{~S}_{\mathrm{Ni}}\right)=52 \mathrm{~J} \cdot \mathrm{K}^{-1} \cdot \mathrm{mol}^{-1}[3,4]$

- Enthalpy of sulfur $h\left(\mathrm{~S}_{\mathrm{Ni}}\right)$ is dependent on surface coverage [2-5]
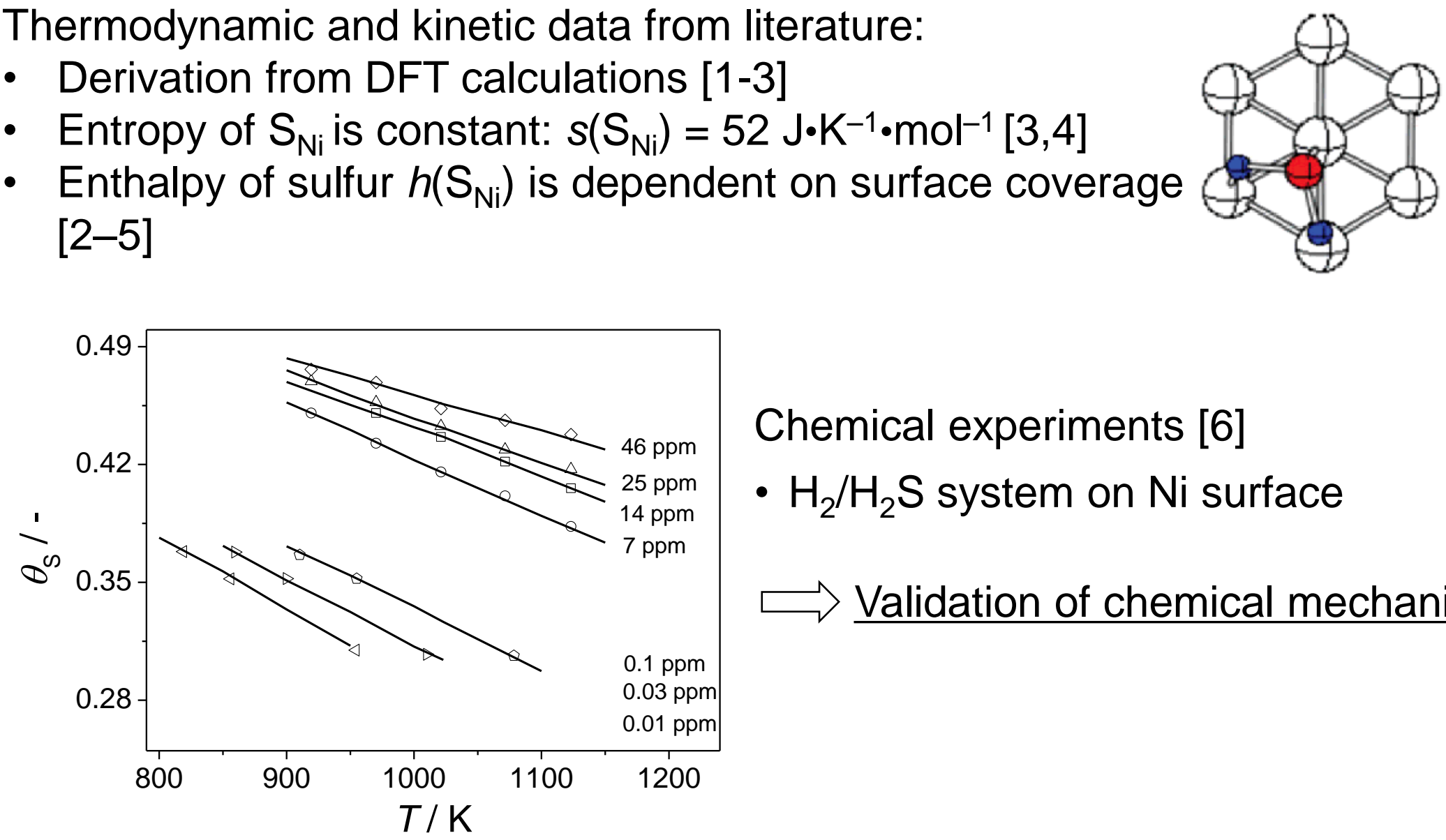

Chemical experiments [6]

- $\mathrm{H}_{2} / \mathrm{H}_{2} \mathrm{~S}$ system on Ni surface

$\Rightarrow$ Validation of chemical mechanism

1. Alfonso, Surface Science, 602, (2008) 2758; 2. Galea, Journal of Catalysis, 263, (2009), 380

3. Monder et al., ECS Transactions, 57, (2013), 2449; 4. McCarty et al., J.Chem.Phys. 72, (1980), 12;

5. Wang et al., Electrochemistry Communications, 9, (2007), 2212; 6. Alstrup et al., Applied Catalysis, 1, (1981), 303 


\section{Reaction mechanism development: Complete mechanism for $\mathbf{S}$ formation and oxidation}

Species, $i$

$h_{i}\left(\mathrm{~kJ} \cdot \mathrm{mol}^{-1}\right) \quad s_{i}\left(\mathrm{~J} \cdot \mathrm{K}^{-1} \cdot \mathrm{mol}^{-1}\right)$

Gas-phase $(T=1023 \mathrm{~K} / T=1073 \mathrm{~K})$

$\begin{array}{cll}\mathrm{H}_{2} \mathrm{~S}_{\text {gas }} & 8.6 / 10.9 & 253.5 / 255.8 \\ \mathrm{H}_{2, \text { gas }} & 21.4 / 22.9 & 166.9 / 168.4 \\ \mathrm{O}_{2, \text { gas }} & 23.5 / 25.3 & 244.4 / 246.0 \\ \mathrm{H}_{2} \mathrm{O}_{\text {gas }} & -241.9 /-212.8 & 233.7 / 235.7 \\ \mathrm{SO}_{2, \text { gas }} & -261.1 /-258.4 & 306.9 / 309.6\end{array}$

Anode surface

$\begin{array}{ccc}\square_{\mathrm{Ni}} & 0 & 0 \\ \mathrm{O}_{\mathrm{Ni}} & -221.6 & 38.9 \\ \mathrm{H}_{\mathrm{Ni}} & -31.8 & 40.7 \\ \mathrm{OH}_{\mathrm{Ni}} & -192.7 & 106.4 \\ \mathrm{H}_{2} \mathrm{O}_{\mathrm{Ni}} & -273.2 & 130.7 \\ \mathrm{H}_{2} \mathrm{~S}_{\mathrm{Ni}} & -43.2 & 0 \\ \mathrm{HS}_{\mathrm{Ni}} & -132.0 & 0 \\ \mathrm{SO}_{2, \mathrm{Ni}} & -486.0 & 0 \\ \mathrm{SO}_{\mathrm{Ni}} & -295.0 & 0 \\ \mathrm{~S}_{\mathrm{Ni}} & \mathrm{f}\left(G_{S}\right) & 52.0\end{array}$

YSZ surface

$\begin{array}{ccc}\square_{\mathrm{YSZ}} & 0 & 0 \\ \mathrm{O}_{\mathrm{YSZ}}^{2-} & -236.4 & 0 \\ \mathrm{H}_{2} \mathrm{O}_{\mathrm{YSZ}} & -273.0 & 97.9 \\ \mathrm{OH}_{\mathrm{YSZ}}^{1-} & -282.5 & 67.0\end{array}$

Bulk species

$\mathrm{O}_{\mathrm{OYSZ}}^{\times}$

$\mathrm{V}_{\mathrm{YSZ}}^{-}$

0
- Compilation of thermodynamic and kinetic data from various literature sources

- Thermodynamic data for 21 species

- Kinetic data for 15 elementary reactions
Reaction

$$
\begin{aligned}
& \text { Ni surface reactions } \\
& \mathrm{H}_{2, \mathrm{~g}}+2 \square_{\mathrm{Ni}} \rightleftharpoons 2 \mathrm{H}_{\mathrm{Ni}} \\
& \mathrm{H}_{2} \mathrm{O}_{\mathrm{g}}+\square_{\mathrm{Ni}} \rightleftharpoons \mathrm{H}_{2} \mathrm{O}_{\mathrm{Ni}} \\
& \mathrm{H}_{\mathrm{Ni}}+\mathrm{O}_{\mathrm{Ni}} \rightleftharpoons \mathrm{OH}_{\mathrm{Ni}}+\square_{\mathrm{Ni}} \\
& \mathrm{H}_{\mathrm{Ni}}+\mathrm{OH}_{\mathrm{Ni}} \rightleftharpoons \mathrm{H}_{2} \mathrm{O}_{\mathrm{Ni}}+\square_{\mathrm{Ni}} \\
& \mathrm{H}_{2} \mathrm{O}_{\mathrm{Ni}}+\mathrm{O}_{\mathrm{Ni}} \rightleftharpoons 2 \mathrm{OH}_{\mathrm{Ni}} \\
& \mathrm{H}_{2} \mathrm{~S}_{\mathrm{g}}+\square_{\mathrm{Ni}} \rightleftharpoons \mathrm{H}_{2} \mathrm{~S}_{\mathrm{Ni}} \\
& \mathrm{H}_{2} \mathrm{~S}_{\mathrm{Ni}}+\square_{\mathrm{Ni}} \rightleftharpoons \mathrm{HS}_{\mathrm{Ni}}+\mathrm{H}_{\mathrm{Ni}} \\
& \mathrm{HS}_{\mathrm{Ni}}+\square_{\mathrm{Ni}} \rightleftharpoons \mathrm{H}_{\mathrm{Ni}}+\mathrm{S}_{\mathrm{Ni}} \\
& \mathrm{SO}_{\mathrm{Ni}}+\square_{\mathrm{Ni}} \rightleftharpoons \mathrm{S}_{\mathrm{Ni}}+\mathrm{O}_{\mathrm{Ni}} \\
& \mathrm{SO}_{\mathrm{Ni}}+\mathrm{O}_{\mathrm{Ni}} \rightleftharpoons \mathrm{SO}_{2, \mathrm{Ni}}+\square \mathrm{Ni} \\
& \mathrm{SO}_{2, \mathrm{Ni}} \rightleftharpoons \mathrm{SO}_{2, \mathrm{~g}}+\square_{\mathrm{Ni}}
\end{aligned}
$$

\begin{tabular}{|c|c|}
\hline $9.80 \cdot 10^{17} \mathrm{~cm}^{4} \cdot \mathrm{mol}^{-2} \cdot \mathrm{s}^{-1}$ & 0 \\
\hline $1.4 \cdot 10^{10} \mathrm{~cm}^{2} \cdot \mathrm{mol}^{-1} \cdot \mathrm{s}^{-1}$ & 0 \\
\hline $5.0 \cdot 10^{22} \mathrm{~cm}^{2} \cdot \mathrm{mol}^{-1} \cdot \mathrm{s}^{-1}$ & 97.9 \\
\hline $3.0 \cdot 10^{20} \mathrm{~cm}^{2} \cdot \mathrm{mol}^{-1} \cdot \mathrm{s}^{-1}$ & 42.7 \\
\hline $5.42 \cdot 10^{23} \mathrm{~cm}^{2} \cdot \mathrm{mol}^{-1} \cdot \mathrm{s}^{-1}$ & 209.4 \\
\hline$s_{i}^{0}=0.5$ & 0 \\
\hline $1.0 \cdot 10^{22} \mathrm{~cm}^{2} \cdot \mathrm{mol}^{-1} \cdot \mathrm{s}^{-1}$ & 14.5 \\
\hline $1.0 \cdot 10^{22} \mathrm{~cm}^{2} \cdot \mathrm{mol}^{-1} \cdot \mathrm{s}^{-1}$ & 10.6 \\
\hline $1.0 \cdot 10^{22} \mathrm{~cm}^{2} \cdot \mathrm{mol}^{-1} \cdot \mathrm{s}^{-1}$ & 158.2 \\
\hline $1.0 \cdot 10^{22} \mathrm{~cm}^{2} \cdot \mathrm{mol}^{-1} \cdot \mathrm{s}^{-1}$ & 61.8 \\
\hline $1.0 \cdot 10^{10} \mathrm{~s}^{-1}$ & 0 \\
\hline $6.595 \cdot 10^{11} \mathrm{~cm}^{2} \cdot \mathrm{mol}^{-1} \cdot \mathrm{s}^{-1}$ & 0 \\
\hline $1.6 \cdot 10^{25} \mathrm{~cm}^{2} \cdot \mathrm{mol}^{-1} \cdot \mathrm{s}^{-1}$ & 164.0 \\
\hline $1.6 \cdot 10^{22} \mathrm{~cm}^{2} \cdot \mathrm{mol}^{-1} \cdot \mathrm{s}^{-1}$ & 91.0 \\
\hline $0.34 \cdot 10^{20} \mathrm{~cm}^{2} \cdot \mathrm{mol}^{-1} \cdot \mathrm{s}^{-1}$ & 181.4 \\
\hline
\end{tabular}

YSZ surface reactions

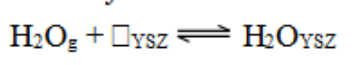$$
\mathrm{H}_{2} \mathrm{O}_{\mathrm{YsZ}}+\mathrm{O}_{\mathrm{YSZ}}^{2-} \rightleftharpoons 2 \mathrm{OH}_{\mathrm{YSZ}}^{1-}
$$$$
\mathrm{O}_{\mathrm{OYSZ}}^{\times}+\square_{\mathrm{YsZ}} \rightleftharpoons \mathrm{V}_{\mathrm{YsZ}}+\mathrm{O}_{\mathrm{YsZ}}^{2-}
$$

Charge-transfer reaction

$\mathrm{H}_{\mathrm{Ni}}+\mathrm{OH}_{\mathrm{YSZ}}^{1-}-\rightleftharpoons \square_{\mathrm{Ni}}+\mathrm{e}^{-}+\mathrm{H}_{2} \mathrm{O}$ YsZ $k^{0}\left(\right.$ or $\left.s_{i}^{0}\right) \quad E^{\text {act }}\left(\mathrm{kJ} \cdot \mathrm{mol}^{-1}\right)$

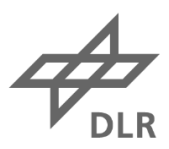




\section{Overview}

$>$ Motivation and aim of the work

$>$ Computational methods

$>$ Results

- Reaction mechanism development

- Sulfur poisoning in $\mathrm{H}_{2} / \mathrm{H}_{2} \mathrm{O}$ fuels

- Sulfur poisoning of internal methane steam reforming

$>$ Summary and conclusions 


\section{Sulfur poisoning in $\mathrm{H}_{2} / \mathrm{H}_{2} \mathrm{O}$ fuels: Impedance modeling}

- Impedance measurements of ASC at $1023 \mathrm{~K}$ and different $i$
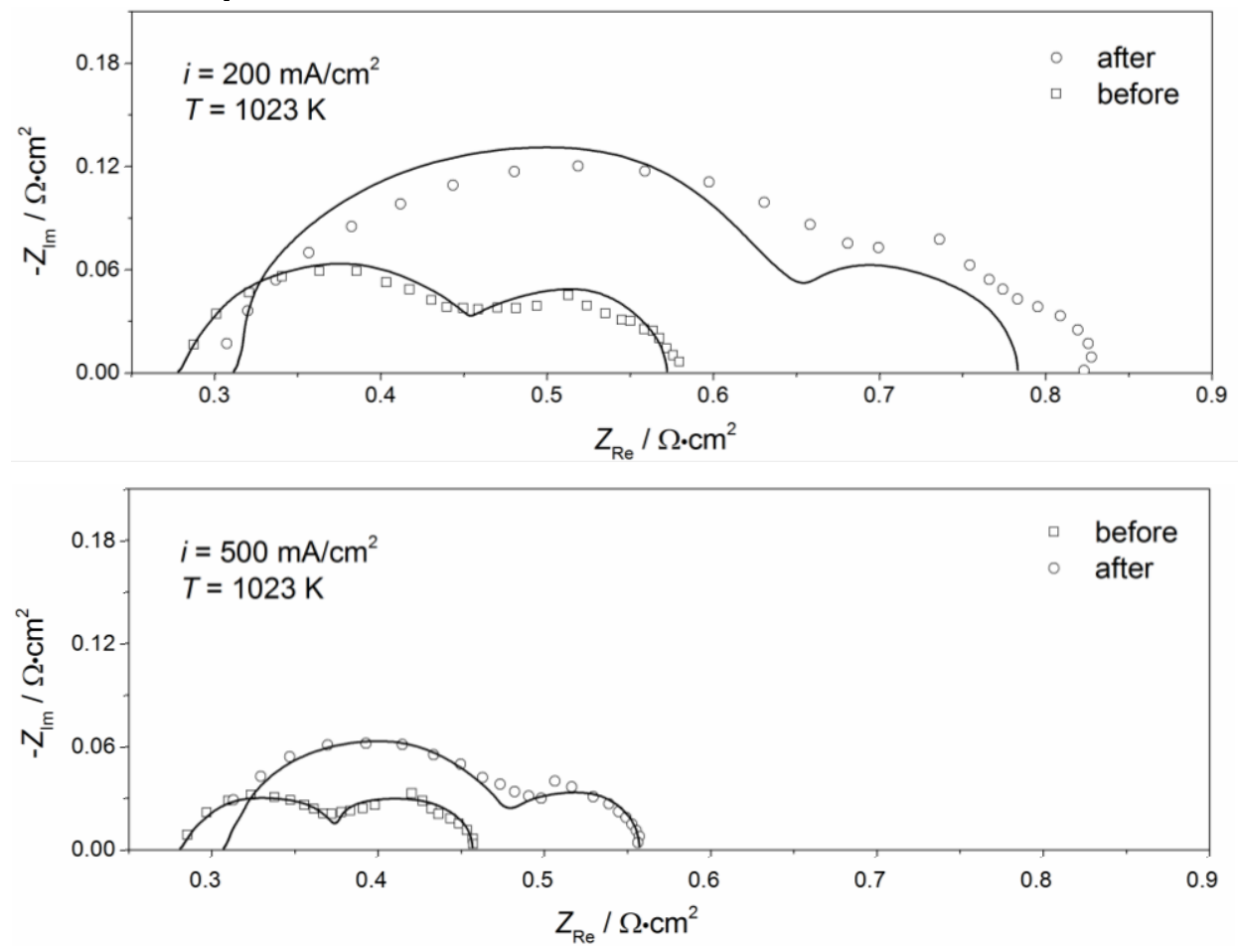

- Gas phase composition: $50 \% \mathrm{H}_{2}, 1.5 \% \mathrm{H}_{2} \mathrm{O}, 48.5 \% \mathrm{~N}_{2}$ 1 ppm $\mathrm{H}_{2} \underline{\mathrm{S}}$

- Good agreement between simulations and experiments for systems with and without sulfur

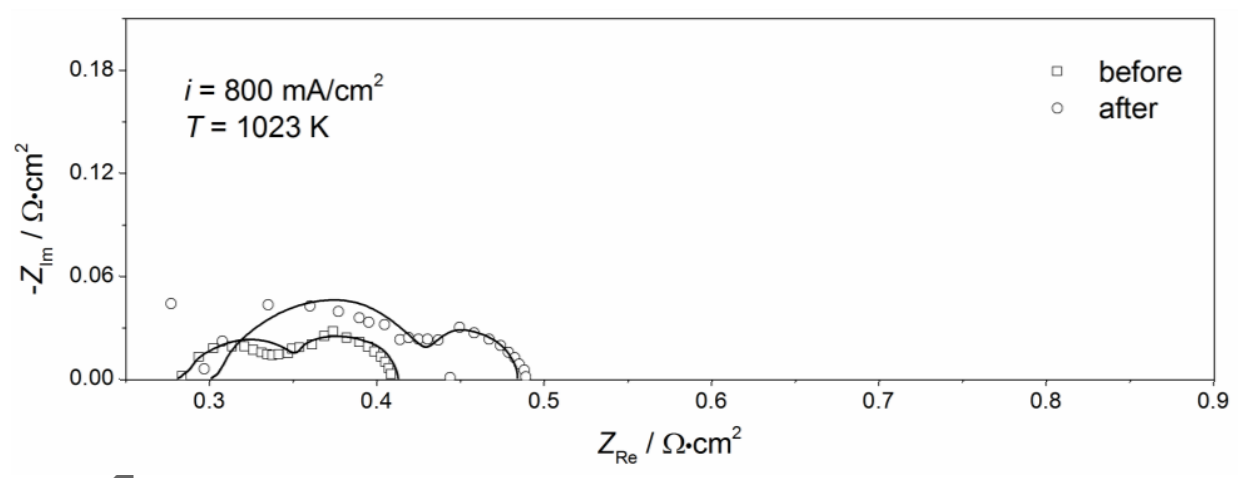

Exp.: Yang et al., Energy \& Environmental Science, 3, (2010) 1804 


\section{Sulfur poisoning in $\mathrm{H}_{2} / \mathrm{H}_{2} \mathrm{O}$ fuels: Performance drops}

- Button cell configuration with $50 \mu \mathrm{m} \mathrm{Ni/YSZ,} 250 \mu \mathrm{m}$ YSZ and $50 \mu \mathrm{m}$ LSM

- Gas composition: $50 \% \mathrm{H}_{2}, 1.5 \% \mathrm{H}_{2} \mathrm{O}, 48.5 \% \mathrm{~N}_{2}, T=1073 \mathrm{~K}$
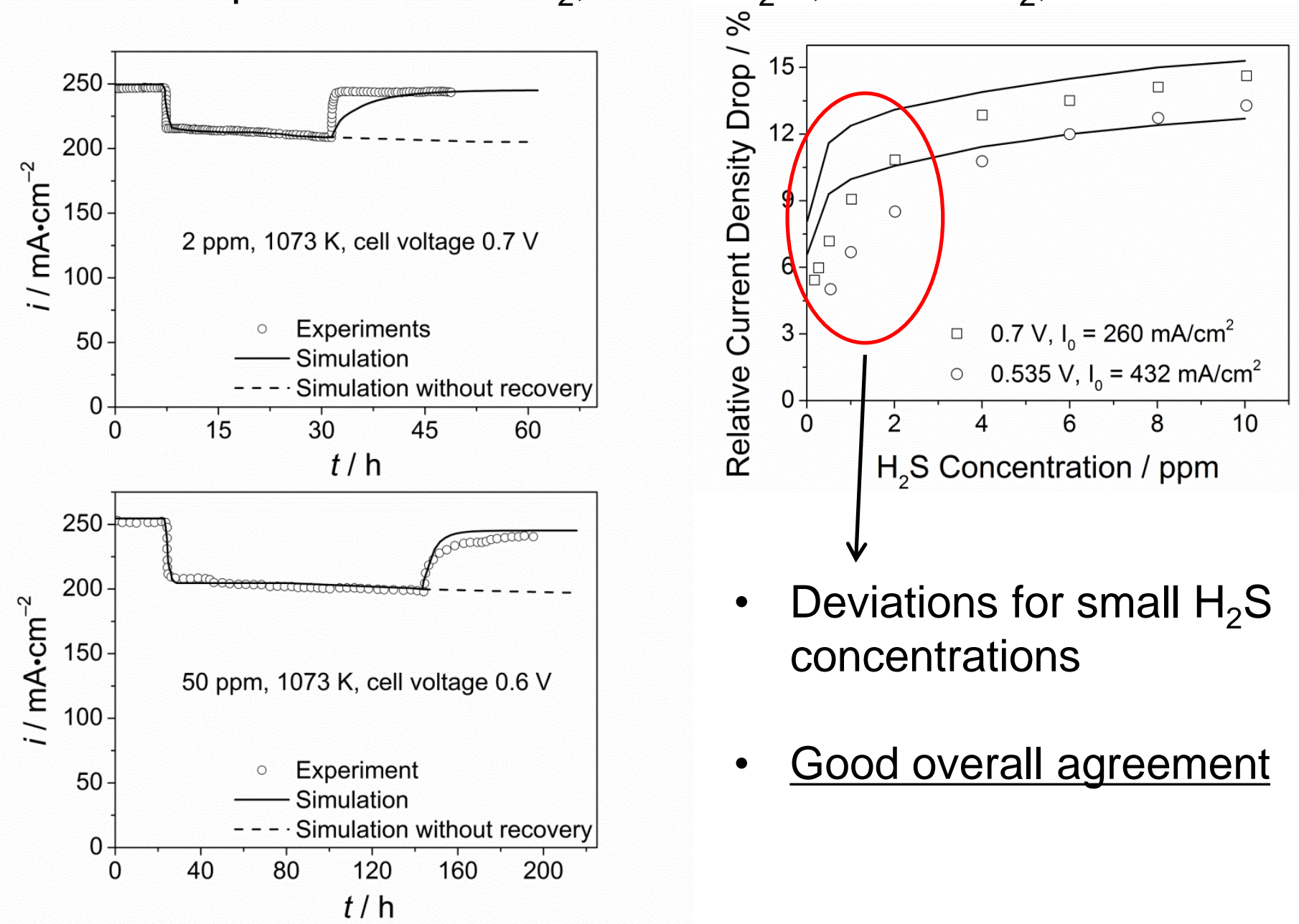

- Deviations for small $\mathrm{H}_{2} \mathrm{~S}$ concentrations

- Good overall agreement 


\section{Sulfur poisoning in $\mathrm{H}_{2} / \mathrm{H}_{2} \mathrm{O}$ fuels: Sulfur coverage}

- Button cell configuration with $50 \mu \mathrm{m} \mathrm{Ni/YSZ,} 250 \mu \mathrm{m}$ YSZ and $50 \mu \mathrm{m}$ LSM

- Gas composition: $50 \% \mathrm{H}_{2}, 1.5 \% \mathrm{H}_{2} \mathrm{O}, 48.5 \% \mathrm{~N}_{2}, T=1073 \mathrm{~K}$

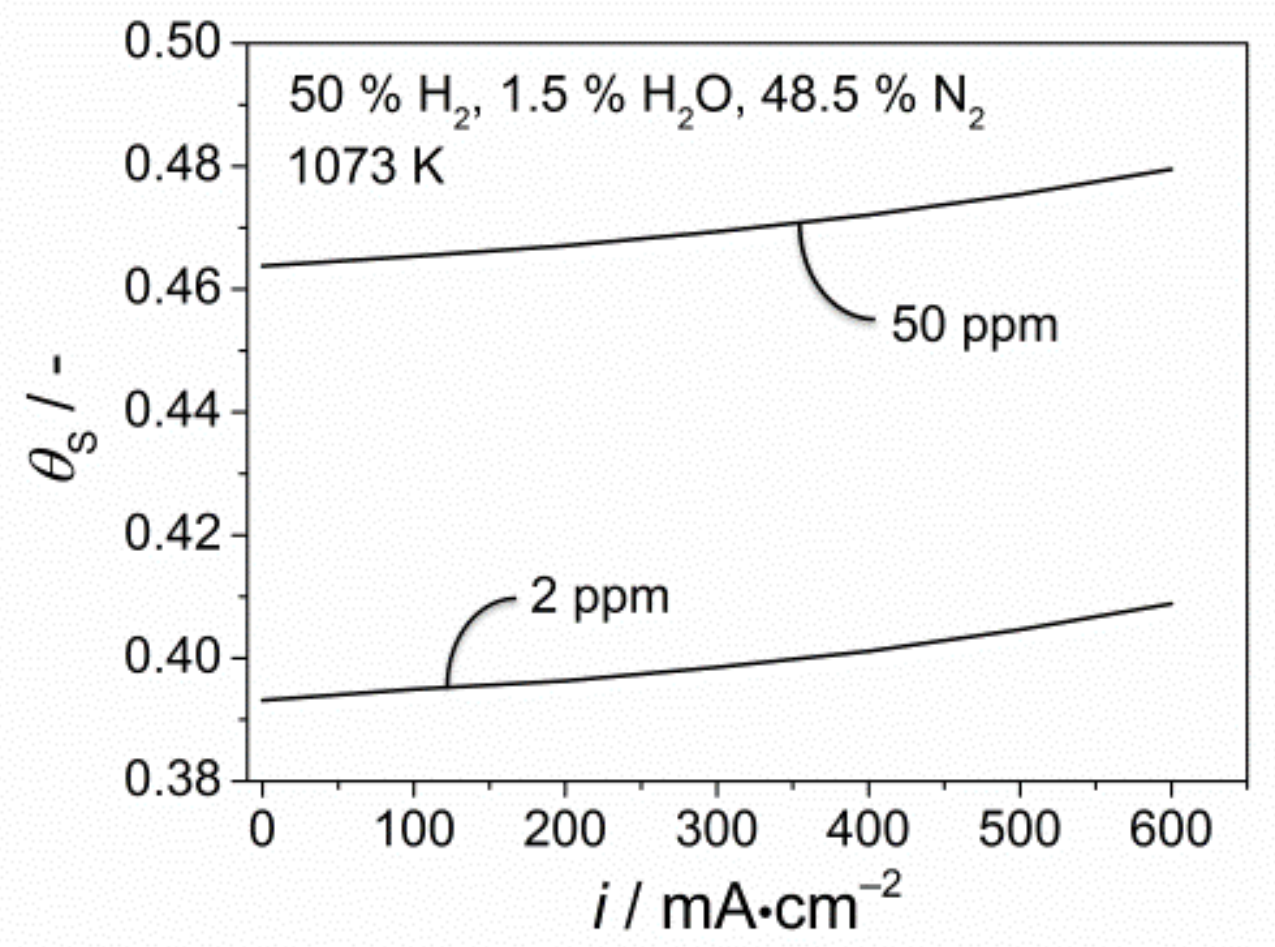

- Literature [1-3]: Decrease of relative polarization resistance with current density

- Suggestion: Electrochemical oxidation of sulfur

$$
\mathrm{S}+2 \mathrm{O}^{2} \Rightarrow \mathrm{SO}_{2, \mathrm{~g}}+4 \mathrm{e}^{-}
$$

- However, sulfur coverage increases with current density 


\section{Overview}

$>$ Motivation and aim of the work

$>$ Computational methods

$>$ Results

- Reaction mechanism development

- Sulfur poisoning in $\mathrm{H}_{2} / \mathrm{H}_{2} \mathrm{O}$ fuels

- Sulfur poisoning of internal methane steam reforming

$>$ Summary and conclusions 


\section{Sulfur poisoning of internal methane steam reforming}

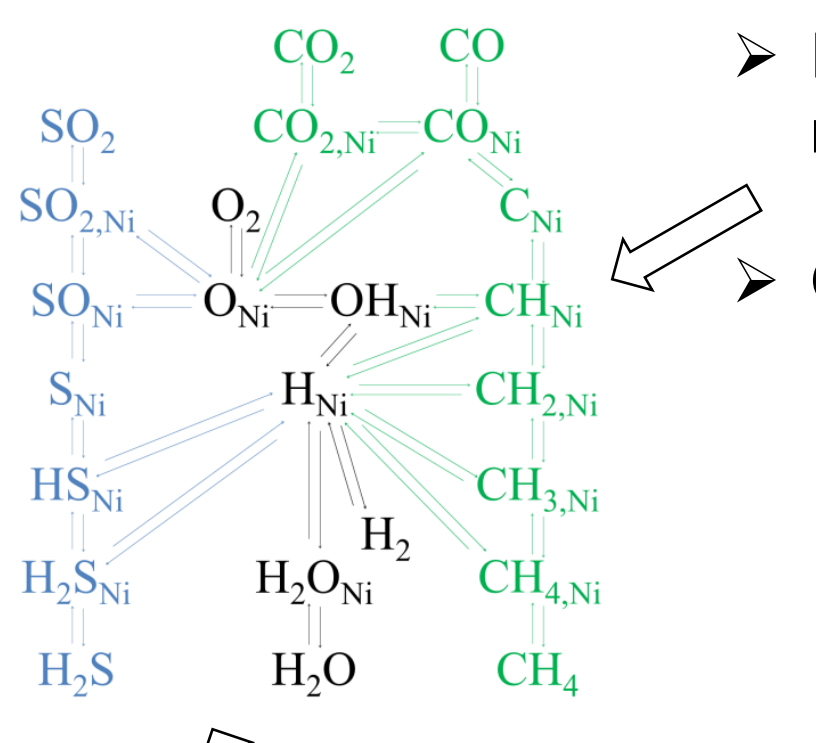

Elementary kinetic reaction mechanism for methane reforming on $\mathrm{Ni} / \mathrm{YSZ}$ [1]

Global reactions:

- Methane steam reforming

$$
\mathrm{CH}_{4}+\mathrm{H}_{2} \mathrm{O} \rightleftharpoons 3 \mathrm{H}_{2}+\mathrm{CO}
$$

- Water gas shift reaction

$$
\mathrm{CO}+\mathrm{H}_{2} \mathrm{O} \rightleftharpoons \mathrm{H}_{2}+\mathrm{CO}_{2}
$$

- Methane dry reforming

$$
\mathrm{CH}_{4}+\mathrm{CO}_{2} \rightleftharpoons 2 \mathrm{H}_{2}+2 \mathrm{CO}_{2}
$$

$>42$ surface reactions, 6 gases, 14 surface

$>$ Reaction mechanism species for sulfur poisoning in $\mathrm{H}_{2} / \mathrm{H}_{2} \mathrm{O}$ systems 


\section{Sulfur poisoning of internal methane steam reforming}

Rasmussen, Hagen [1]:

- Sulfur poisoning of methane steam reforming in ASC at $T=850^{\circ} \mathrm{C}$

- Transient poisoning experiments at OCV
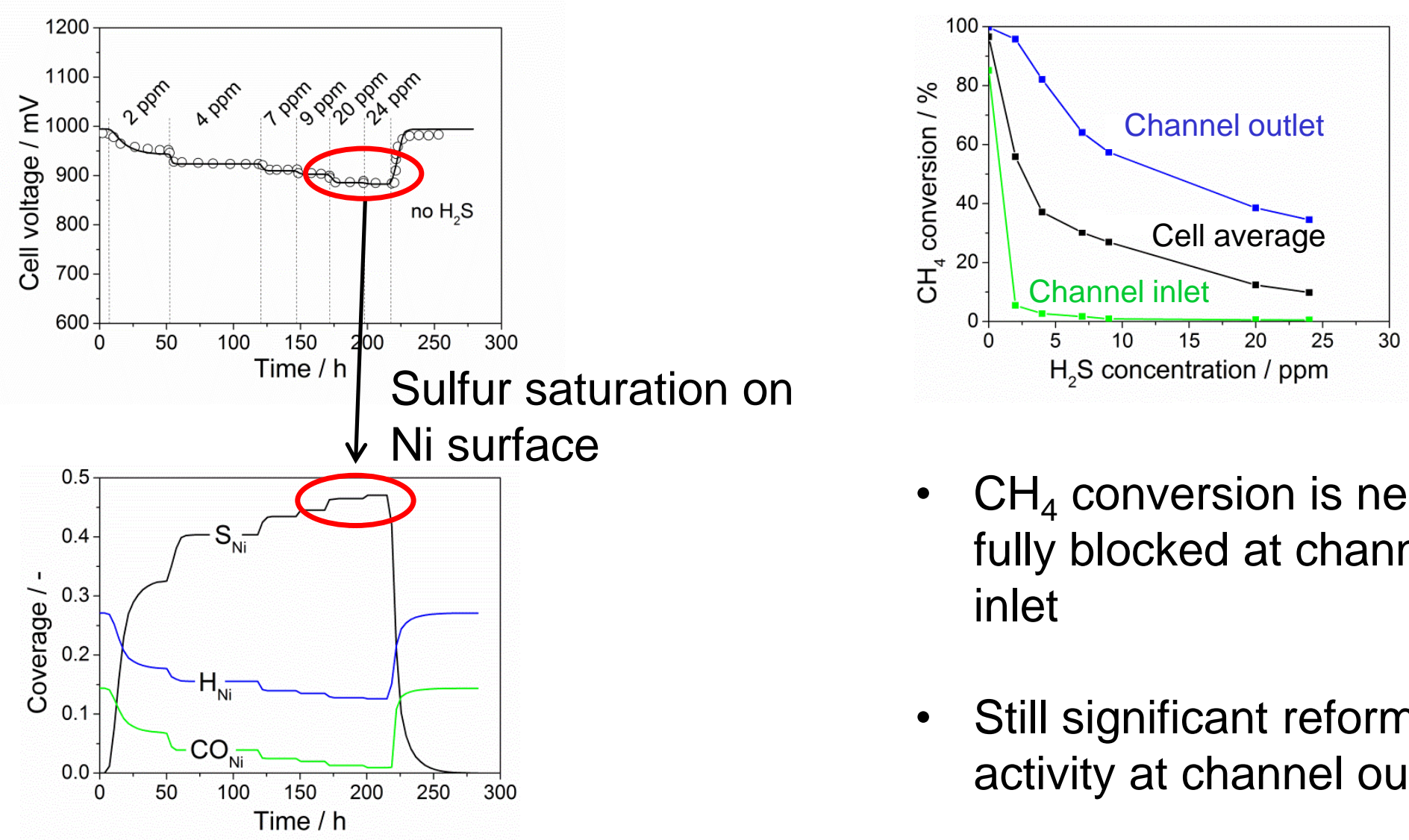

- $\mathrm{CH}_{4}$ conversion is nearly fully blocked at channel inlet

- Still significant reforming activity at channel outlet 


\section{Sulfur poisoning of internal methane steam reforming}

- Transient poisoning experiments under current load $\left(i=1 \mathrm{~A} \cdot \mathrm{cm}^{-2}\right)$

- Impedance spectroscopy measurements
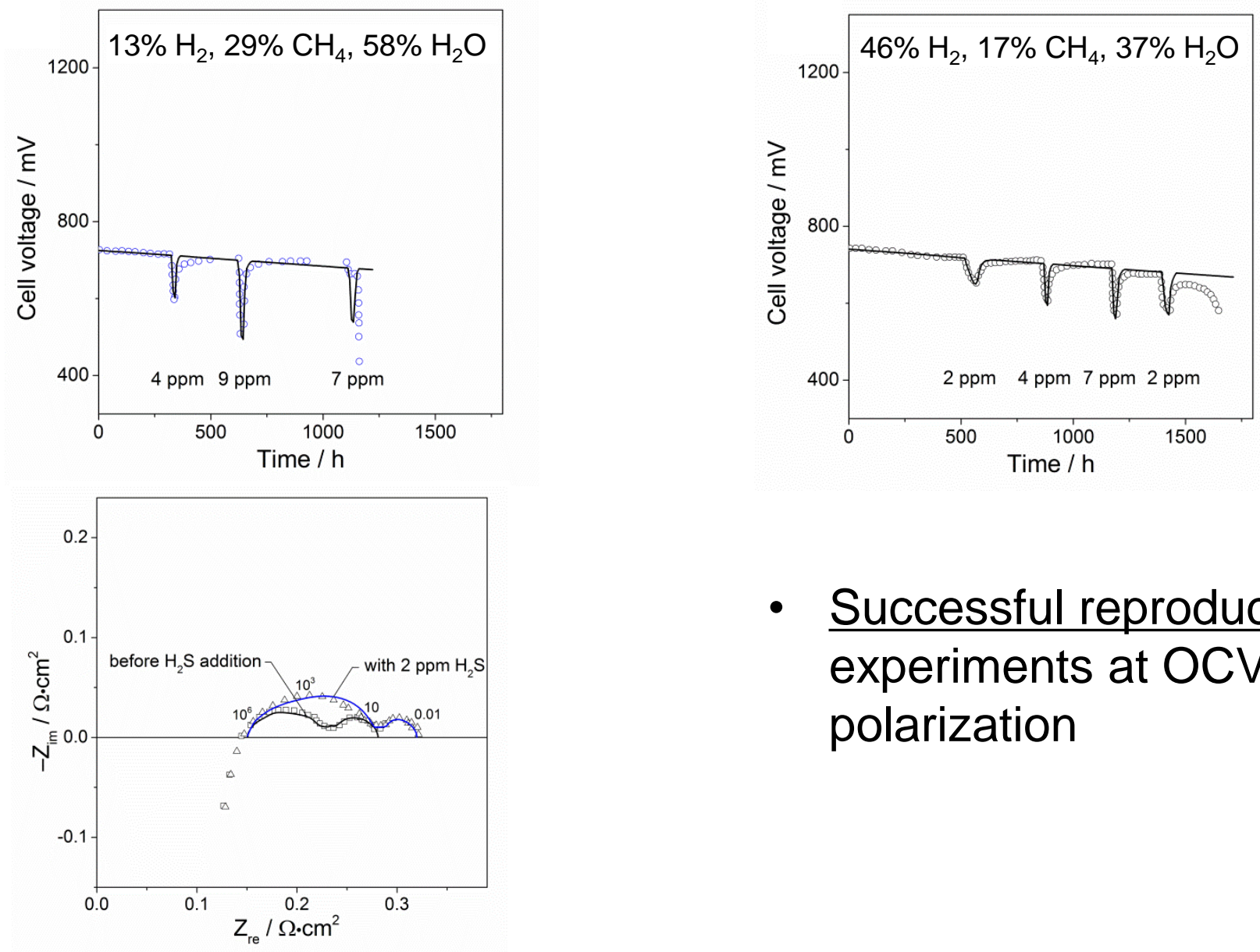

- Successful reproduction of experiments at OCV and under polarization 


\section{Sulfur poisoning of internal methane steam reforming: Gas phase species distribution}

Detailed analysis: Gas phase distribution along the channel $T=850{ }^{\circ} \mathrm{C} ; i=1 \mathrm{~A} \cdot \mathrm{cm}^{-2} ; 13 \% \mathrm{H}_{2}, 29 \% \mathrm{CH}_{4}, 58 \% \mathrm{H}_{2} \mathrm{O}$

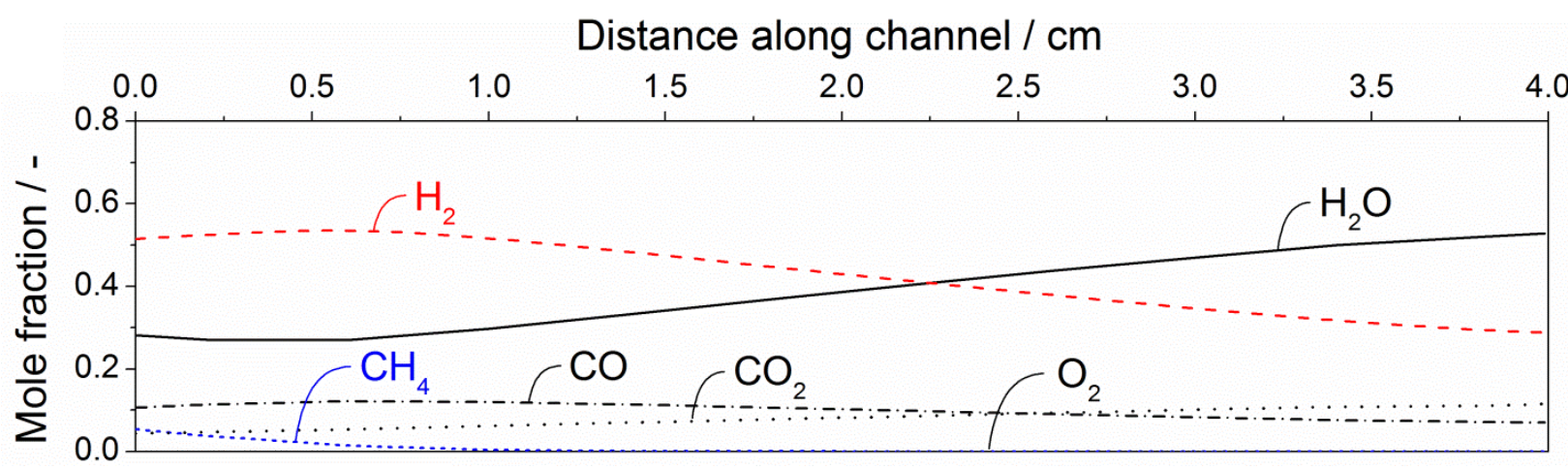

- Without $\mathrm{H}_{2} \mathrm{~S}$

- Rapid $\mathrm{CH}_{4}$ reforming

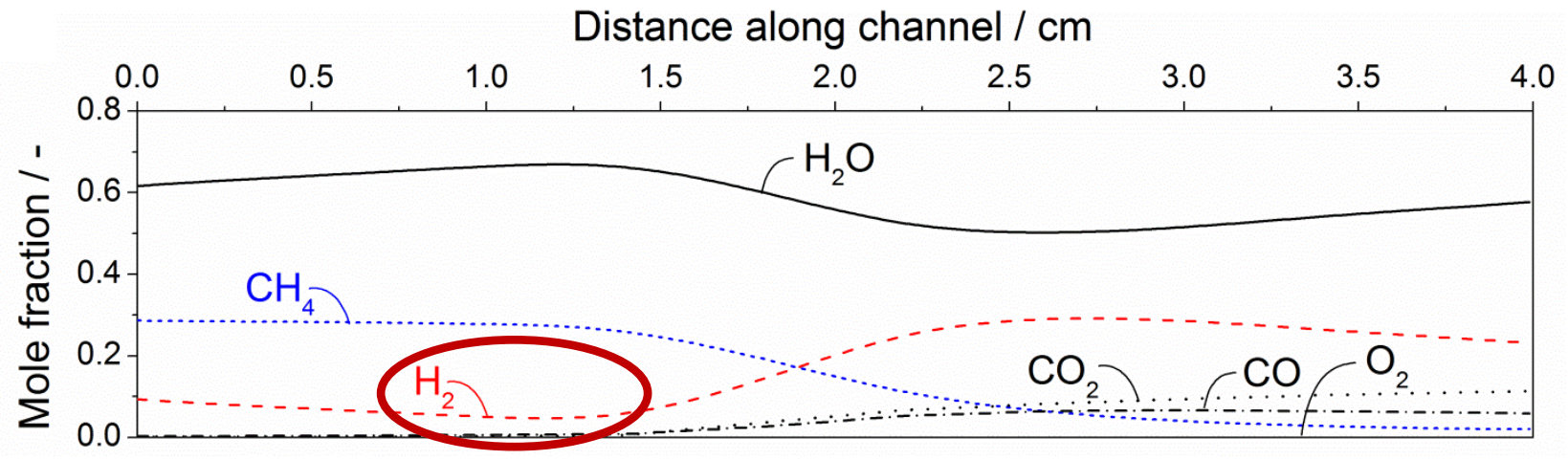

- $2 \mathrm{ppm} \mathrm{H} \mathrm{H}_{2} \mathrm{~S}$

- Danger of fuel starvation 


\section{Overview}

$>$ Motivation and aim of the work

$>$ Computational methods

$>$ Results

- Reaction mechanism development

- Sulfur poisoning in $\mathrm{H}_{2} / \mathrm{H}_{2} \mathrm{O}$ fuels

- Sulfur poisoning of internal methane steam reforming

$>$ Summary and conclusions 


\section{Summary and conclusions}

- An elementary kinetic reaction mechanism has been developed and validated for sulfur poisoning of $\mathrm{Ni} / \mathrm{YSZ}$ anodes

- $\mathrm{H}_{2} / \mathrm{H}_{2} \mathrm{O}$ systems

- Internal methane steam reforming

- A thermodynamic and kinetic data set has been compiled

- Sulfur coverage increases with increasing current density

- Own experiments are required for more profound investigation 


\section{Acknowledgments}

- German network project: "SOFC Degradation"

- Funding by Germany Ministry of Education and Research (BMBF) via grant number 03SF0494C

Thank you for your attention!

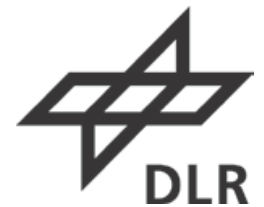

Bundesministerium für Bildung und Forschung

\section{\#raunhofer} IKTS

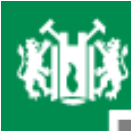

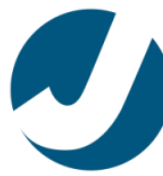

JÜLICH

FORSCHUNGSZENTRUM
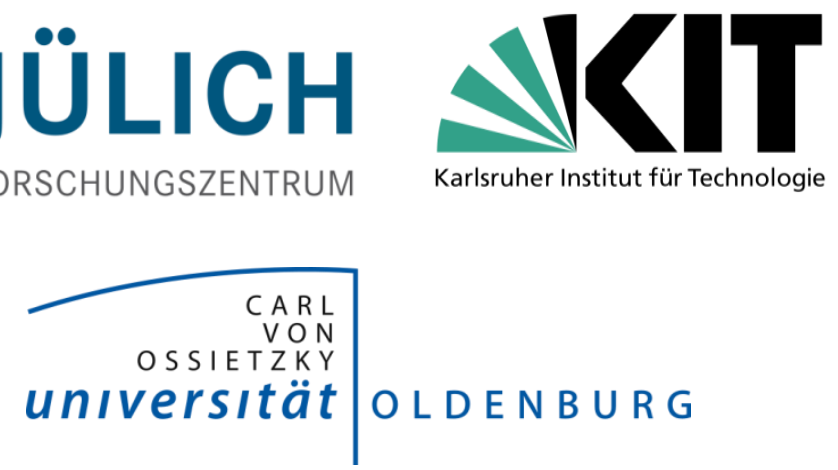

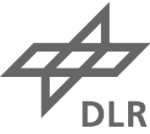

